\title{
ESBL Positive Organisms: Method of Routine Reporting and Prevalence in Health Care Settings.
}

\author{
Shah Md Zahurul Haque Asna ${ }^{1}$, Shameem Akhter ${ }^{1}$, M Mushfequr Rahman ${ }^{1}$, Najib Mohammad ${ }^{2}$, M A Hafez $^{3}$ \\ ${ }^{1}$ Department of Microbiology, Bangladesh University of Health Sciences (BUHS), ${ }^{2}$ Department of Intensive Care Medicine \\ Bangladesh Institute of Health Sciences \& Hospital (BIHSH), ${ }^{3}$ Department of Epidemiology \& Biostatistics Bangladesh \\ University of Health Sciences (BUHS)
}

\begin{abstract}
Extended Spectrum B- Lactamase (ESBL) acquired by bacteria is a highly effective weapon for drug resistance against a wide range of antibiotics (penicillins, cephalosporins and monobactams). ESBLs have continued to increase in variety and prevalence and are now a global health concern including Bangladesh. They are associated with failure in effective treatment, increased morbidity and mortality, poor outcomes, increases in length of stay(LOS) in hospital and health care costs. So, it is important to identify it and take necessary measures to treat with appropriate antimicrobial therapy. This study has been designed to establish an easy method for routine reporting of ESBL organisms and notify it's incidence in Health Care settings. Double Disk Diffusion Test, utilizing Cefotaxime, Amoxicillin/Clavulanic acid and Ceftazidime, was used as Screening Test. Phenotypic Confirmatory Test was done for confirmation of screening ESBL positive organisms. Four hundred seventy two organisms (Escherichia coli and Klebsiella sp.) were isolated from urine and pus collected from Health Care settings. Only 50 screening ESBL positive organisms and 50 ESBL negative organisms were tested by Phenotypic Confirmatory Test. All of them were positive and negative respectively by Phenotypic Confirmatory Test. Predominant organism among the isolates was Escherichia coli $(\mathbf{8 2 . 8} \%)$ of which $\mathbf{5 4 . 4 \%}$ were ESBL positive followed by Klebsiella sp. $(\mathbf{1 7 . 2 \%})$ of which $\mathbf{4 4 . 4 \%}$ were ESBL positive. ESBL positivity was more in hospital infections $(\mathbf{7 3 . 3 \% )}$ than in community acquired infections $(\mathbf{4 4 . 5 \% )}$.
\end{abstract}

Key words: ESBL, Drug resistance.

\section{Introduction:}

Resistant bacteria are emerging world wide in community and health care settings specially in Intensive Care Units ${ }^{1}$. Extended Spectrum $\beta$ - Lactamase(ESBL) is the latest addition to this. The ESBL isolates were first reported from Germany and England in $1983^{2,3}$ and subsequently in USA in late $1988^{3,4}$. In Bangladesh, it has been reported in $2001^{5}$.

ESBLs are enzymes that mediate resistance to ß-lactam drugs, viz. penicillins, cephalosporins and monobactams but do not affect cephamycins or carbapenems. Because of greatly extended substrate range, these enzymes are called

\section{Correspondence:}

Prof. Shah Md Zahurul Haque Asna

Head, Department of Microbiology,

Bangladesh University of Health Sciences (BUHS)

125/1 Darussalam, Mirpur, Dhaka-1216, Bangladesh

Cell: 01817-510403

Email:asnabd04@yahoo.com extended spectrum $\beta$-lactamases ${ }^{1}$,. ESBL enzymes are most commonly produced by Escherichia coli (E. coli) and Klebsiella $s p$ and to some extent by other members of Enterobacteriaceae and Pseudomonadaceae ${ }^{1,6,7}$.

ESBLs have continued to increase in variety (more than 400 variants detected $)^{8}$ and prevalence and are now a global health concern ${ }^{3,9}$. The ESBLs have implications for clinicians and patients because they are associated with failure of effective treatment, increased morbidity and mortality, poor outcomes, increased length of stay (LOS) in hospital and health care costs ${ }^{9}$.

So, it is important to identify it early and properly and take necessary measures to treat with appropriate antibiotics. In developing countries like Bangladesh, its reporting with routine sensitivity tests is not widely practiced. Aim of this study was to i) establish an easy routine detection method so that every laboratory can identify and report ESBL positive 
organisms and ii) notify its prevalence in Health Care Settings.

\section{Materials and methods}

This is a cross-sectional study. This study was done in the Department of Microbiology, Bangladesh Institute of Health Sciences (BIHS), Dhaka, Bangladesh from March, 2012 to February, 2013. Samples (urine and pus) were collected from BIHS hospital, it's satellite hospitals and out door settings located in and around Dhaka city. Approval of ethical committee was waived because the samples were received in laboratory and contact with patients was not necessary.

Four hundred seventy two organisms isolated from urine and pus were studied. Pus was collected from diabetic foot lesions, post surgical infected wounds and traumatic wounds. Only E. coli and Klebsiella $s p$. were included in this study and other organisms were excluded due to their small number(about 2\%). Isolation and identification of the organisms was done by standard procedures ${ }^{10,11}$.

Media for sensitivity: Muller Hinton agar(Oxoid) ${ }^{12,13}$. Antibiotic discs were placed on Muller Hinton agar according to standard procedure ${ }^{13}$. (Figure.I)

\section{Detection of ESBL activity:}

For detection of ESBL positive organisms, screening test ${ }^{14,15}$ was done along with routine sensitivity test. Positive screening tests were confirmed later by Phenotypic Confirmatory Test (PCT) ${ }^{12}$.

\section{Screening test:}

Double disk diffusion test (DDDT) was used as screening test. Disks of Cefotaxime (CTX), Ceftazidime (CAZ) and Amoxycillin/ Clavulonic acid (AMC) were placed in a line, placing $\mathrm{AMC}$ in the centre and other two on either side as shown in Fig.I. Distance between AMC and other two were $25 \mathrm{~mm}$ from centre to centre. Other antibiotic disks were placed as standard procedure in the same plate along with screening test for ESBL activity. Widening of cephalosporin's inhibition zone adjacent to the disk containing clavulonic acid (AMC) was regarded as ESBL positive ${ }^{13,14,15}$ (Figure.I).

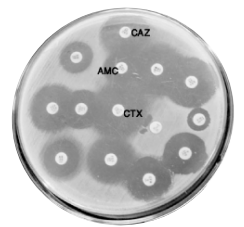

A.

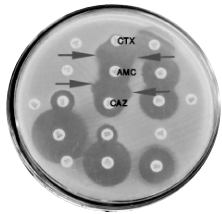

B.
Fig. I. Shows routine antibiotic sensitivity test together with screening test(DDDT). A.: ESBL negative;

B: ESBL positive(arrow indicates widening of zone of inhibition)

\section{Phenotypic Confirmatory test (PCT):}

In this method, two sets were used. One set consists of Ceftazidime $(30 \mu \mathrm{g})$ disk and Ceftazidime/ Clavulanic acid $(30 / 10 \mu \mathrm{g})$ disk. Another set consists of Cefotaxims $(30 \mu \mathrm{g})$ disk and Cefotaxims / Clavulonic acid $(30 / 10 \mu \mathrm{g})$. The disks among the sets were placed not less than $30 \mathrm{~mm}$ apart (centre to centre). When the zone of inhibition by combination disks (Ceftazidime /Clavulanic acid or Cefotaxime/ Clavulonic acid) was $\geq 5 \mathrm{~mm}$ than the zone of inhibition produced by Ceftazidime /Cefotaxime alone, the test was taken as PCT positive. Only 50 Screening ESBL positive and 50 Screening ESBL negative organisms were tested by PCT. E.coli ATCC ${ }^{\circledR} 25922$ was used as control organism for both sets of PCT. In an ideal setting, the zone of inhibition by the Ceftazidime /Cefotaxime alone is $\leq 2 \mathrm{~mm}$ less than the zone of inhibition produced by combination disks ${ }^{12}$.

\section{Results:}

\section{Identification of ESBL Positive organisms:}

Prevalence of ESBL organisms:

Table I. shows the incidence of ESBL positive organisms. Out of 472 organisms studied, 249(52.8\%) were ESBL positive by ESBL screening test. Out of these, 50 ESBL Screening test positive and $50 \mathrm{ESBL}$ screening negative organisms were tested by PCT, all (100\%) of which were found positive and negative respectively. It was also noted that E.coli $(82.8 \%)$ was more prevalent than Klebsiella $s p$. (17.2\%) and ESBL positivity was more in E.coli $(54.5 \%)$ than in case of Klebsiella (44.4\%).

Distribution of ESBL positive organisms in hospital admitted patients (IPD) and out patients (OPD) has been shown in Table. II. Isolation of ESBL positive organism was higher in samples taken from patients admitted in hospital $(73.3 \%, \mathrm{n}=135)$ than samples from out-patient departments $(44.5 \%, \mathrm{n}=337)$.

Table I. Result of Screening and Phenotypic confirmatory test (PCT) for ESBL activity

\begin{tabular}{l|c|c|c}
\hline Name of test & $\begin{array}{c}\text { Name of } \\
\text { Organisms tested }\end{array}$ & $\begin{array}{c}\text { Result: } \\
\text { N (\%) of } \\
\text { ESBL Positive } \\
\text { organisms }\end{array}$ & Total N(\%) \\
\hline $\begin{array}{l}\text { Screening test } \\
(\text { DDDT })\end{array}$ & E. coli $(N=391)$ & $213(54.5)$ & \multirow{2}{*}{$249(52.8)$} \\
\cline { 2 - 3 } $\begin{array}{l}\text { Phenotypic* } * \\
\text { confirmatory } \\
\text { test(PCT) }\end{array}$ & $\begin{array}{c}\text { ESBL Screening } \\
\text { positive N=50 }\end{array}$ & $36(44.4)$ & $50(100)$ \\
\cline { 2 - 3 } & $\begin{array}{c}\text { Screening ESBL } \\
\text { Negative N=50 }\end{array}$ & $0(0)$ & \multirow{2}{*}{$0(0)$} \\
\hline
\end{tabular}

Note: * for PCT, both E.coli and Klebsiella sp. were studied proportionately 
Table II: Distribution of ESBL + ve organisms in hospital admitted patients (IPD) and out patients (OPD).

\begin{tabular}{lcccc}
\hline Organism & \multicolumn{2}{c}{ IPD } & \multicolumn{2}{c}{ OPD } \\
\cline { 2 - 5 } & $\begin{array}{c}\text { Number } \\
\text { studied }\end{array}$ & $\begin{array}{c}\text { ESBL+ } \\
\text { N(\%) }\end{array}$ & $\begin{array}{c}\text { Number } \\
\text { studied }\end{array}$ & $\begin{array}{c}\text { ESBL+ } \\
\text { N(\%) }\end{array}$ \\
\hline Esch. coli & 100 & $76(76)$ & 291 & $137(47.1)$ \\
Klebseilla & 35 & $23(65.7)$ & 46 & $13(28.3)$ \\
Total & 135 & $89(65.9)$ & 337 & $150(44.5)$ \\
\hline
\end{tabular}

\section{Discussion:}

ESBL producers are associated with increased morbidity and high mortality rate, increased length of stay (LOS) in hospital and increased health care costs. Not only in hospitals, they are also emerging in community ${ }^{1,9}$. Routine reporting of ESBL positive organisms with their antibiotic sensitivity is essential for proper treatment of the patients. This study was designed to develop a system to routinely report ESBL positive organisms and to high light the latest scenario of prevalence in health settings located in and around Dhaka city.

For detection of ESBL positive organisms all authors screened the isolates first by a test called screening test and later on confirmed by one or more confirmatory tests $3,12,16,17,18,19,20$. The method used for screening in this study is called Double Disk Diffusion Test (DDDT) as described by other authors ${ }^{14,15}$. Screening positive isolates were subjected to Phenotypic Confirmatory Test (PCT) as recommended by the Clinical Laboratory Standard Institution (CLSI) ${ }^{12}$. However, most of the authors used CLSI guide line (Disk diffusion /agar dilution method) ${ }^{12}$ for screening ESBL positive organisms where reduced susceptibility to any of Ceftriaxone, Cefotaxime and Ceftazidime was taken as screening ESBL positive $e^{3,16,17,20,21}$. These were further confirmed by one or more of DDDT (also Called DDST) ${ }^{18,20 \text {, }}$ PCT ${ }^{16,19,}$ E -test ${ }^{3,16,17}$ and/or PCR ${ }^{19,20,21}$ by different authors. Dalela (2012) ${ }^{16}$ used CLSI criteria of diminished zone of disk diffusion for screening ESBL positive organisms and used DDST (DDDT), PCT and E- test as confirmatory test. By DDDT, $57.5 \%(\mathrm{n}=126)$ and by PCT, 61.6\% $(\mathrm{n}=135)$ of screening ESBL positive organisms were confirmed as ESBL producers. E-test correlated with PCT. Isolates were screened by CLSI guide line and confirmed by E-test; $76 \%(n=145)$ of the screening ESBL positives were found as confirmed ESBL positive by E-test ${ }^{17}$.

In this study, 50 ESBL screening positive (by DDDT) organisms and 50 ESBL screening negative organisms were tested by PCT where all (100\%) of screening ESBL positive organisms and Screening ESBL negative organisms were found positive and negative respectively. This showed that the screening test (DDDT) was $100 \%$ sensitive and specific.
E.coli $\mathrm{ATCC}^{\circledR} 25922$ was used as control and tested by PCT which showed $\leq 2 \mathrm{~mm}$ less zone of inhibition by single disk (Ceftazidime or Cefotaxime) than produced by combination disk (Ceftazidime/Clavulonic acid or Cefotaxime/Calvulonic acid $)^{12}$. This indicated that the procedure adopted in this study was on right track.

In our study, Screening Test (DDDT) was done along with routine sensitivity test. Reasons of selecting DDDT as routine ESBL screening test and doing along with routine sensitivity test, were - i) it's sensitivity and specificity was comparable to that of PCT and $\mathrm{E}-$ test $^{16}$; ii) The test is very simple and can be done along with routine sensitivity test. Only appropriate positioning of three disks (Ceftazidime, Cefotaxime, and Amoxycillin/Clavulonic acid) has to be made. iii) no extra cost and iv) saves 24 hours than the method recommended by $\mathrm{CLSI}^{12}$.

In this study, 472 organisms were tested of which $249(52.8 \%)$ were ESBL positive. This correlates with findings of Ahmed et al $(53.4 \%, \mathrm{n}=221)^{18}$ and Sashirekha $(48.9 \%, \mathrm{n}=225)^{17}$. Findings of Dalela $(61.6 \%, \mathrm{n}=219)^{16}$, Oliveira et al $(61.1 \%, \mathrm{n}=90)^{19}$ and $\mathrm{Yasmin}^{21},(71.3 \%, \mathrm{n}=300)$ were slightly higher; the findings of Rahman et $a l^{5}$ was slightly lower(41.4\%, $\mathrm{n}=157)$, but that of Hansen et al $(2.4 \%$, $\mathrm{n}=14674)^{7}$ was much lower. It is to be noted that in countries where there is enhanced infection control practices and antimicrobial stewardship, the prevalence of ESBL is lower than other countries as was evidenced in a study in 2009 where global prevalence rate was found as follows: However, Prevalence of ESBL positive organisms are increasing throughout the world ${ }^{3}$.

Predominating organism in our study was E.coli $(82.8 \%$, $\mathrm{n}=472)$ followed by Klebsiella sp. $(17.2 \%$,) of which $54.5 \%$ $(\mathrm{n}=391)$ of E.coli and $44.4 \%(\mathrm{n}=81)$ of Klebsiella $s p$. were ESBL Positive. This finding correlates with study of Rahman et $a l^{5}$ where predominating organism was E.coli $(54.4 \%$, $\mathrm{n}=241$ ), of which $43.2 \%$ were ESBL positive, followed by K.pneumoniae (45.6\%) of which $39.5 \%$ were ESBL positive. In the study of Sashirekha (2013) $)^{17}$ predominating organism was E. coli $(28 \%, \mathrm{n}=325)$, of which $52.8 \%$ was ESBL positive, followed by K.pneumoniae (25.2\%) of which $45.1 \%$ was ESBL positive. In the study of Dalela (2012) ${ }^{16}$ like ours predominating organism was E.coli $(50.7 \%, \mathrm{n}=219)$ followed by K.pneumoniae (19.6\%) but prevalence of ESBL positivity was higher, than ours, where E. coli $(73.5 \%)$ was predominating ESBL producer followed by K.pneumoniae (58.1\%). In contrast, study of Olveira et al (2010) ${ }^{19}$ showed K.pneumoniae $(71.1 \%, \mathrm{n}=90)$ as predominating organism 
followed by Escherichia coli (24.4\%) of which $71.9 \%$ of K.pneumoniae and $36.4 \%$ of E.coli were ESBL positive. Study of Yasmin $(2012)^{21}$ in Bangladesh also showed different scenario than ours where predominating organism was E. coli $(52 \%, \mathrm{n}=300)$ followed by Proteus $s p .(18.3 \%$, and Klebsiella sp. $(15 \%$,$) ; in her study, Klebsiella sp (80\%)$ topped the list as ESBL producers followed by Proteus $s p$ (72\%) and E.coli (67.3\%).

When comparison was made between incidence of ESBL positive organisms in hospital admitted patients (hospital acquired) and out- patients (which may also be called community acquired), the incidence amongst hospital admitted patients $(73.3 \%, \mathrm{n}=135)$ was found significantly $(\mathrm{P}<0.001)$ higher than out- patients $(44.5 \%, \mathrm{n}=337)$. However, it is a matter of Public Health concern that large number of ESBL positive organisms are being community acquired (i.e.patients attending out-patient or out -door health care settings). This correlates with study of Ahmed et al (2010) ${ }^{18}$ where 84(71.2\%)) ESBL producers were hospital acquired and $34(28.8 \%)$ from out patients(community). This contrasts with findings of Yasmin $(2012)^{21}$ who found more ESBLs in community $(58.4 \%, \mathrm{n}=214)$ than in hospital $(41.6 \%)$.

\section{Conclusion:}

Prevalence of ESBL positive organisms in health care settings and community level is quite high. They can be easily identified by screening test using common antibiotic disks in routine sensitivity tests. While reporting the culture and sensitivity tests, the ESBL positive organisms should be pointed out with comment; The organism is ESBL Positive; which is resistant to Penicillins, Cephalosporins and Monobactams.

\section{Reference:}

1. Chaudhary U and Aggarwal R. Extended spectrum Blactamases(ESBL)- an emerging threat to clinical tharapies. Ind J Med Microbiol.2004;22(2):75-80.

2. Knothe H, Shah P,Kremery V et al. Transferable resistance to cefotaxime, cefoxitin, cefomandole and cefuroxime in clinical isolates of Klebsiella pneumonia and Serratia marsescens. Infection 1983;11:315-7.

3. Rupp M E and Fey P D. Extended spectrum B-lactamase (ESBL)- producing Enterobacteriaceae. Drugs 2003;63(4):353-365.

4. Jacoby JA. Extended spectrum ß-lactamases and other enzymes providing resistance to oxyimino ß-lactams. Infect Dis Clin North Am 1997;11:875-87
5. Rahman MM et al. Prevalence of extended spectrum ?lactamase-producing Escherichia coli and Klebsiella pneumonia in an urban hospital in Dhaka, Bangladesh. International Journal of antimicrobial agents. 2004; 24: 508-510.

6. Dudely MN, Ambrose PG, Bhavnani SM et al. Background and rationale for revised Clinical and Laboratory Standards Institute interpretive criteria (breakpoints) for Enterobacteriaceae and Pseudomonas aeroginosa:I. Cephalosporins and astreonam. Clin Infect Dis 2013;56:1301-9.

7. Hansen DS, Schumacher H, Hansen $\mathrm{F}$ et al. Extended spectrum B-lactamase(ESBL) in Danis clinical isolates of Escherichia coli and Klebsiella pneumonia: prevalence, ß-lactamase distribution, phylogroups and co-resistance. Scan J Infect Dis 2012 Mar;44(3): 174-81.

8. Lahey. B-Lactamase Classification and Amino Acid Sequences for TEM, SHV and OXA Extended-Spectrum and Inhibitor Resistant Enzymes. http://www.lahey.org/studies/ (14 june 2013, date last accessed).

9. Fennell et al. Increasing prevalence of ESBL production among Irish clinical Enterobacteriaceae from 2004 to 2008: an observational study. BMC Infectious Diseases. 2012; 12:116 -123.

10. Nataro JP et al. Escherichia,Shigella and Salmonella. In: Versalovic J, Carrol KC, Funke G,Jorgensen JH, Landry ML, Warnock DW(eds).Manual of Clinical Microbiology. Volume 1, 10th edition.ASM press, American Society for Microbiology, Washington DC. 2011.p.603-6024

11. Abbott S.L.Klebsiella, enterobacter, Citrobacter, Serratia Plesiomonas and other. In: Versalovic J, Carrol KC, Funke G,Jorgensen JH, Landry ML, Warnock DW(eds). Manual of Clinical Microbiology. Volume 1, 10th edition. ASM press, American Society for Microbiology, Washington DC. 2011.p.639-657.

12. CLSI. Performance Standard for Antimicrobial Susceptibility Testing; Twenty -second Information Supplement. M100S22, Vol.32 No.3, 2012, January.p.20-60.

13. NCCLS. Performance Standards for Antimicrobial Disk Susceptibility Tests-Sixth edition; Approved standard. 1997 January. M2-A6,Vol.17 No.1. p.5-17

14. Jarlier V, Nicolas MH, Fournier G, Philoppon A. Extended broad -spectrum beta-lactamases conferring transferrable resistance to newer beta-lactam agents in 
Enterobacteriaceae: hospital prevalence and susceptibility pattern. Rev Infect Dis. Jul-Aug. 1988;10(4):867-78.

15. Miles RS and Amyes SGB. Laboratory control of antimicrobial therapy.In: Collee JG, Fraser AG, Marmion BP,Simmons A(eds.). Mackie and McCartney Practical Medical Microbiology. Fourteenth Edition. Churchill Livingstone. 1999. p.169.

16. Dalela G. Prevalence of Extended Spectrum Beta Lactamase(ESBL) producers among Gram Negative Bacilli from Various Clinical Isolates in a tertiary Care Hospital at Jhalawar, Rajasthan, India. Journal of Clinical and Diagnostic research. April. 2012;6(2):182-187.

17. Sasirekha B. Prevalence of ESBL, AMPC B-Lactamases and MRSA Among uropathogens and Its Antibiogram. EXCLI Journal 2013;12 : 81-88.

18. Ahmed K, Thokar MA, Toboli AS et al. Extended spectrum $ß$-lactamase mediated resistance in Escherichia coli in a tertiary care hospital in Kashmir, India. Afr. J.Microbiol. Res.2010; 4(24) : 2720-2728.

19. Oliveira CFD, Salla A, Lara VM et al.Prevalence of Extended -Spectrum- Beta- Lactamases-Producing Microorganisms in Nosocomial Patients and Molecular Characterization of the SHV Type Isolates. Brazilian Journal of Microbiology 2010;41:278-282.

20. Jonathan N.Screening for Extended - Spectrum Betalactamase- Producing Pathogenic Enterobacteria in District General Hospitals. J Clin Microbiol. Mar 2005;43(3):1488-1490.

21. Yasmin T. Prevalence of ESBL among Esch. coli and Klebsiella spp. in a tertiary care hospital and molecular detection of important ESBL producing genes by multiplex PCR..M.Phil thesis. Department of Microbiology, Mymemsing Medical College, Mymensingh 2012 January: p. 6,86,89 